\title{
COMUNICAÇÃO
}

\section{AVALIAÇÃO DA EFICIÊNCIA DOS FUNGICIDAS FLUDIOXONIL + METALAXYL-M NO TRATAMENTO DE SEMENTES DE SORGO ${ }^{1}$}

\author{
Evaluation of the efficiency of the fungicides \\ fludioxonil + metalaxyl-M in the treatment of sorghum seeds
}

\author{
Nicésio Filadelfo Janssen de Almeida Pinto ${ }^{2}$
}

\begin{abstract}
RESUMO
Conduziu-se este trabalho com o objetivo de avaliar a eficiência dos fungicidas fludioxonil + metalaxyl-M no controle de fungos associados às sementes de sorgo da cultivar BR 506 e na proteção delas contra fungos do solo. Assim, sementes de sorgo foram tratadas com os seguintes fungicidas (dose em g i.a./100 kg sementes): fludioxonil + metalaxyl-M $(2,5+1,0)$, fludioxonil + metalaxyl-M (3,75 + 1,5), fludioxonil + metalaxyl-M incolor $(2,5+1,0)$, fludioxonil + metalaxyl-M incolor $(3,75+1,5)$ e thiram $(140,0)$. Sementes sem tratamento fungicida constituíram a testemunha. Foram avaliadas as seguintes características: sanidade das sementes (papel de filtro com congelamento), emergência de plântulas em solo esterilizado (casa-de-vegetação), emergência em solo com monocultivo de sorgo (casa-de-vegetação) e emergência no teste de frio em solo com monocultivo de sorgo (laboratório e casa-de-vegetação). Pelas análises dos resultados (Tukey a $5 \%$ ), constatou-se que: 1- O fungicida thiram foi eficiente no controle de Fusarium subglutinans associado às sementes de sorgo, porém, ineficiente no controle de Aspergillus spp.; 2- Os fungicidas fludioxonil + metalaxyl-M, fludioxonil + metalaxyl-M incolor e thiram foram eficientes no controle de Penicillium spp., Alternaria tenuis, Phoma sorghina, Curvularia lunata e Drechslera turcica; 3- Todos os tratamentos fungicidas das sementes incrementaram, em solo esterilizado, a emergência de plântulas de sorgo devido ao controle dos fungos patogênicos associados às sementes, e 4- Todos os tratamentos fungicidas incrementaram, em solo com monocultivo de sorgo, a emergência de plântulas, pela proteção das sementes de sorgo contra fungos de solo.
\end{abstract}

Termos para indexação: Sorghum bicolor, fungos, patologia de sementes, emergência de plântulas.

\begin{abstract}
This work was carried out in order to evaluate the efficiency of several fungicides applied as seed dressing in the control of sorghum seedborne fungi and the protection against soilborne fungi. Sorghum seeds of cultivar BR 506 were treated with the following fungicides (doses in $\mathrm{g}$ a.i./100 $\mathrm{kg}$ of seeds): fludioxonil + metalaxyl-M $(2.5+1.0)$, fludioxonil + metalaxyl-M $(3.75+1.5)$, colorless fludioxonil + metalaxyl-M $(2.5+1.0)$, colorless fludioxonil + metalaxyl-M $(3.75+1.5)$ and thiram (140.0). Seeds without fungicide treatment constituted the check. The following characteristics were evaluated: seed health (deepfreezing method), seedling emergence in sterile soil (green house), seedling emergence in sorghum monocropped soil (green house) and cold test in sorghum monocropped soil (laboratory and green house). The analyses of the results (Tukey at 5\%) showed that: 1- the fungicide thiram was effective in the control of Fusarium subglutinans associated to the sorghum seeds, but ineffective against Aspergillus spp.; 2fungicides fludioxonil + metalaxyl-M, colorless fludioxonil + metalaxyl-M and thiram were effectively in the controlled Penicillim spp., Alternaria tenuis, Phoma sorghina, Curvularia lunata and Drechslera turcica; 3- all fungicides had a positive effect on seedling emergence due to the protection against pathogenic fungi associated to sorghum seeds, and 4- all the fungicide treatments increased the seedling emergence in sorghum monocropped soil due to protection of seeds against soilborne fungi.
\end{abstract}

Index terms: Sorghum bicolor, fungi, seed pathology, seedling emergence.

(Recebido para publicação em 26 de maio de 2003 e aprovado em 3 de outubro de 2003)

\footnotetext{
1. Trabalho de cooperação entre a Embrapa Milho e Sorgo e a Syngenta.

2. Engenheiro Agrônomo, Pesquisador da Embrapa Milho e Sorgo - Rod. MG 424, Km 65, Caixa Postal 151, 35701-970 - Sete Lagoas, MG. nicesio@cnpms.embrapa.br
} 
A cultura do sorgo está sujeita ao ataque de um número elevado de doenças, cujos patógenos são, na maioria, transmitidos por sementes (BAIN, 1950; HARRIS e LUTTRELL, 1955; MINUSSI, 1977).

A redução da qualidade fisiológica das sementes de sorgo é função direta da presença de fungos patogênicos a elas associadas (LASCA et al., 1986) e, entre outros danos às plantas de sorgo, podem promover decréscimo no rendimento de grãos. As sementes contaminadas constituem-se em fonte de inóculo primário para a doença no campo, como no caso de Phoma e Colletotrichum (MINUSSI e KIMATI, 1978).

O tratamento fungicida é uma das principais medidas recomendadas para o controle de patógenos veiculados por sementes (NEERGAARD, 1979). Apesar de a literatura relatar alguns trabalhos sobre o controle de fungos em sementes de sorgo (HARRIS e LUTTRELL, 1955; HEPPERLY et al., 1982; NOVO e MENEZES, 1984), poucas são as informações específicas a esse respeito. No Brasil, entre os tratamentos testados por Valarini et al. (1988) para o controle de fungos associados às sementes de sorgo, com destaque para Colletotrichum graminicola, os mais eficientes foram carbendazin + thiram, captafol, iprodione + thiram, captafol + PCNB, captan, guazatine + imazalil, benomyl e thiram. Com relação à proteção de sementes de sorgo contra o ataque de fungos do solo, os fungicidas captan e metalaxyl foram eficientes no controle de Pythium sp., ao passo que o thiram e o captan destacaram-se no controle de Rhizoctonia solani (PINTO, 1998).

Este trabalho foi realizado com o objetivo de avaliar a eficiência dos fungicidas fludioxonil + metalaxyl$\mathrm{M}$ no tratamento de sementes de sorgo, visando ao controle de fungos associados às sementes e à proteção contra os fungos de solo Fusarium moniliforme e Pythium aphanidermatum.

Foi utilizada uma amostra de sementes de sorgo da cultivar BR 506, a qual foi subdividida em subamostras de 1,0 kg de sementes, sendo a seguir submetidas aos seguintes tratamentos fungicidas ( dose em $\mathrm{g}$ i.a./100 kg sementes): fludioxonil + metalaxyl-M (2,5 + $1,0)$, fludioxonil + metalaxyl-M $(3,75+1,5)$, fludioxonil + metalaxyl-M incolor $(2,5+1,0)$, fludioxonil + metalaxyl-M incolor $(3,75+1,5)$ e thiram $(140,0)$. Sementes sem tratamento fungicida constituíram a testemunha.

Para os tratamentos fungicidas (6 tratamentos em 4 repetições), as sementes foram acondicionadas em sacos plásticos e umedecidas com água destilada $(0,75 \%$ p/v). Após a aplicação, as sementes foram agi- tadas até a perfeita homegeneização dos fungicidas nas mesmas.

Para avaliar o desempenho do tratamento fungicida sobre a micoflora das sementes, dos fungos do solo e a emergência de plântulas, foram realizados os seguintes testes:

1. Análise de sanidade das sementes: empregou-se o método do papel de filtro com congelamento (LIMONARD, 1966), em que as sementes acondicionadas em caixa gerbox contendo 3 folhas de papel de filtro embebidos em água foram colocadas inicialmente por 24 horas em câmara de incubação regulada em 22 $\pm 2 \mathrm{C}$ e sob regime de 12 horas de luz e 12 horas de escuro, sendo, em seguida, submetidas ao congelamento (-20 C) por 24 horas. Findo esse tempo, as sementes foram retornadas para a câmara de incubação, onde permaneceram por mais 5 dias para o adequado desenvolvimento dos fungos que infectam ou infestam as sementes de sorgo. Após a incubação, as sementes foram examinadas sob microscópio estereoscópico (50 aumentos) para a identificação e a quantificação dos fungos associados às sementes. O delineamento experimental foi o inteiramente ao acaso, com 6 tratamentos em 4 repetições de 100 sementes.

2. Emergência em solo esterilizado: este teste foi realizado para se avaliar a eficiência dos fungicidas testados no controle de fungos associados às sementes de sorgo. O substrato de germinação foi constituído da mistura de solo de cultivo e areia fina lavada (1:1), esterilizado a $120^{\circ} \mathrm{C} / 2$ horas, e acondicionado em caixas de metalon de $50 \times 30 \times 10 \mathrm{~cm}$. Cada caixa possuía 12 divisões (células), e 100 sementes de sorgo foram semeadas em cada célula, perfazendo o total de 300 sementes por repetição de tratamento. A umidade do substrato ideal para a germinação foi mantida pela adição de volumes controlados de água destilada. Imediatamente após a semeadura, as caixas com as sementes foram colocadas em casa-de-vegetação regulada em 25 $\pm 5^{\circ} \mathrm{C}$. Decorridos 10 dias da semeadura, procedeu-se à avaliação da porcentagem de plântulas de sorgo emergidas (PINTO, 1997). O delineamento experimental foi o inteiramente ao acaso com 6 tratamentos em 4 repetições.

3. Emergência de plântulas em casa-devegetação em solo com monocultivo de sorgo: por meio deste teste avaliou-se a eficiência dos fungicidas testados, no controle dos fungos de solo na semeadura e daqueles associados às sementes. Foi utilizado substrato de germinação, acondicionado em caixa de metalon, constituído pela mistura de solo de área com monocul- 
tivo de sorgo e areia fina lavada (2:1). O solo estava naturalmente infectado com fungos patogênicos à cultura do sorgo, principalmente por Fusarium moniliforme e Pythium aphanidermatum. A semeadura e a condução desse teste foram realizadas como descrito para o teste de emergência de plântulas em solo esterilizado. Decorridos 10 dias da semeadura, procedeu-se à avaliação da porcentagem de plântulas emergidas. $\mathrm{O}$ delineamento experimental foi o inteiramente casualizado, com 6 tratamentos em 4 repetições.

4. Emergência no teste de frio em solo de campo: com este teste avaliou-se a eficiência dos fungicidas no controle de fungos do solo, patogênicos às sementes de sorgo, em condições favoráveis à germinação. Solo de área com monocultivo de sorgo naturalmente infestado com fungos patogênicos às sementes foi utilizado como substrato de germinação, para estudar a interação fungos de solo versus tratamento fungicida das sementes. $\mathrm{O}$ solo foi acondicionado em caixas de metalon, as quais foram semeadas como descrito no ítem 2 . O delineamento experimental foi o inteiramente ao acaso, com 6 tratamentos em 4 repetições. Após a semeadura, as sementes foram colo- cadas por 7 dias em incubadora regulada em $10 \pm 1^{\circ} \mathrm{C}$. A seguir, as caixas com as sementes foram transferidas para casa-de-vegetação regulada em $25 \pm 5^{\circ} \mathrm{C}$, onde, após 10 dias, foi avaliada a porcentagem de plântulas emergidas (PINTO, 1997).

Os resultados obtidos estão contidos na Tabela 1 e mostram que: 1- $\mathrm{O}$ fungicida thiram foi eficiente no controle de Fusarium subglutinans associado às sementes de sorgo, porém, ineficiente no controle de Aspergillus spp.; 2- Os fungicidas fludioxonil + metalaxyl-M, fludioxonil + metalaxyl-M incolor e thiram foram eficientes no controle de Penicillium spp., Alternaria tenuis, Phoma sorghina, Curvularia lunata e Drechslera turcica; 3- Todos os tratamentos fungicidas das sementes incrementaram em solo esterilizado (ESE) a emergência de plântulas de sorgo devido ao controle dos fungos patogênicos associados às sementes, e 4- Todos os tratamentos fungicidas incrementaram em solo com monocultivo de sorgo (ETFSC e ETFSC) a emergência de plântulas pela proteção das sementes de sorgo contra os fungos de solo Fusarium moniliforme e Pythium aphanidermatum.

TABELA 1 - Microbiota das sementes e emergência de plântulas oriundas de sementes de sorgo da cultivar BR 506 tratadas com fungicidas. Embrapa Milho e Sorgo, Sete Lagoas, MG, 2001.

\begin{tabular}{|c|c|c|c|c|c|c|c|c|c|c|c|}
\hline & \multirow{2}{*}{ Dose $^{1}$} & \multicolumn{7}{|c|}{ Microbiota das sementes (\%) } & \multicolumn{3}{|c|}{ Emergência de plântulas (\%) } \\
\hline & & $\mathbf{F S}^{2}$ & $\mathbf{P E N}^{3}$ & $\mathbf{A S P}^{4}$ & $\mathbf{A T}^{5}$ & $\mathbf{P H}^{6}$ & $\mathbf{C U R}^{7}$ & DRE $^{8}$ & ESE $^{9}$ & ESCCV $^{10}$ & ETFSC $^{11}$ \\
\hline Testemunha & 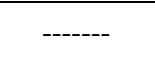 & $61,8 \mathrm{a}^{12}$ & $8,0 \mathrm{a}$ & $11,9 \mathrm{~b}$ & $15,3 \mathrm{a}$ & $44,5 \mathrm{a}$ & $2,5 \mathrm{a}$ & $1,4 \mathrm{a}$ & $66,0 \mathrm{~b}$ & $61,2 \mathrm{~b}$ & $17,0 \mathrm{~b}$ \\
\hline $\begin{array}{l}\text { Fludioxonil + } \\
\text { Metalaxyl-M }\end{array}$ & $2,5+1,0$ & $75,1 \mathrm{a}$ & $0,4 \mathrm{~b}$ & $2,4 \mathrm{~b}$ & $0,6 \mathrm{~b}$ & $1,9 \mathrm{~b}$ & $0,3 \mathrm{~b}$ & $0,2 \mathrm{~b}$ & $74,7 \mathrm{a}$ & $75,1 \mathrm{a}$ & $68,4 \mathrm{a}$ \\
\hline $\begin{array}{l}\text { Fludioxonil + } \\
\text { Metalaxyl-M }\end{array}$ & $3,75+1,5$ & $78,6 \mathrm{a}$ & $0,3 \mathrm{~b}$ & $4,1 \mathrm{~b}$ & $0,4 \mathrm{~b}$ & $1,6 \mathrm{~b}$ & $0,2 \mathrm{~b}$ & $0,5 \mathrm{ab}$ & $76,3 \mathrm{a}$ & $73,0 \mathrm{a}$ & $68,1 \mathrm{a}$ \\
\hline $\begin{array}{l}\text { Fludioxonil + } \\
\text { Metalaxyl-M } \\
\text { incolor }\end{array}$ & $2,5+1,0$ & $72.1 \mathrm{a}$ & $0,1 \mathrm{~b}$ & $2,2 \mathrm{~b}$ & $1,7 \mathrm{~b}$ & $2,7 \mathrm{~b}$ & $0,5 \mathrm{~b}$ & $0,7 \mathrm{ab}$ & $77,7 \mathrm{a}$ & $73,2 \mathrm{a}$ & $68,6 \mathrm{a}$ \\
\hline $\begin{array}{l}\text { Fludioxonil + } \\
\text { Metalaxyl-M } \\
\text { incolor }\end{array}$ & $3,75+1,5$ & $79,9 \mathrm{a}$ & $0,0 \mathrm{~b}$ & $2,2 \mathrm{~b}$ & $0,6 \mathrm{~b}$ & $1,9 \mathrm{~b}$ & $0,4 \mathrm{~b}$ & $0,3 \mathrm{~b}$ & $74,6 \mathrm{a}$ & $74,9 \mathrm{a}$ & $71,7 \mathrm{a}$ \\
\hline Thiram & 140,0 & $11,3 \mathrm{~b}$ & $0,3 \mathrm{~b}$ & $55,5 \mathrm{a}$ & $0,5 \mathrm{~b}$ & $0,3 \mathrm{~b}$ & $0,1 \mathrm{~b}$ & $0,0 \mathrm{~b}$ & $78,3 \mathrm{a}$ & $74,3 \mathrm{a}$ & 58,9 a \\
\hline C. V. $(\%)$ & & 15,6 & 70,7 & 46,4 & 38,8 & 65,6 & 67,9 & 83,9 & 4,3 & 5,1 & 10,1 \\
\hline
\end{tabular}

${ }^{1}$ Gramas do ingrediente ativo/100 kg de sementes, ${ }^{2}$ Fusarium subglutinans, ${ }^{3}$ Penicillium spp., ${ }^{4}$ Aspergillus spp., ${ }^{5}$ Alternaria tenuis, ${ }^{6}$ Phoma sorghina, ${ }^{7}$ Curvularia lunata, ${ }^{8}$ Drechslera turcica. ${ }^{9}$ Emergência de plântulas em solo esterilizado, ${ }^{10}$ Emergência de plântulas em casa-de-vegetação em solo oriundo de campo com momocultivo de sorgo, ${ }^{11}$ Emergência de plântulas no teste de frio em solo de campo com monocultivo de sorgo. ${ }^{12}$ Numa coluna, as medias seguidas pelas mesmas letras não diferem entre si (Tukey $5 \%$ )

Ciênc. agrotec., Lavras, v. 28, n. 2, p. 450-453, mar./abr., 2004 
Com relação à emergência de plântulas em solo esterilizado (ESE), ficou evidenciada a patogenicidade desses fungos sobre as sementes, sendo as melhores emergências obtidas nos tratamentos com fungicidas. $\mathrm{O}$ incremento da emergência de plântulas é um efeito indireto do fungicida, pois o tratamento fungicida não visa a aumentar a viabilidade da semente, mas se a redução na emergência for causada por fungos, o tratamento eficiente proporcionará incremento dessa característica, como foi observado neste trabalho. Harris e Luttrell (1955) encontraram aumento significativo da emergência de plântulas de sorgo quando as sementes foram tratadas com thiram, o mesmo acontecendo nos trabalhos de Hepperly et al. (1982), Brancão et al. (1983) e Novo e Menezes (1984), que obtiveram resultados semelhantes quando as sementes foram tratadas com thiram e captan. Valarini et al. (1988) infestaram sementes de sorgo com diversos fungos, entre eles Colletotrichum graminicola, porém, não observaram correlação positiva entre os diversos fungicidas utilizados no tratamento dessas sementes e a emergência de plântulas em condição de casa-de-vegetação. Por outro lado, em relação aos fungos do solo, a emergência de plântulas em solo de campo em condição de casa-de-vegetação (ESCCV) e emergência no teste de frio em solo de campo (ETFSC) foi melhor em sementes tratadas com fungicidas.

\section{AGRADECIMENTOS}

A Osni Alves da Silva, Ademar Verneque e José Moreira Campos (Assistentes de Pesquisa), a Ana Luísa Paiva Amaral (Acadêmica de Ciências Biológicas, Faculdades Metodistas Integradas Izabela Hendrix, Belo Horizonte, MG) e a Gilberto Ribeiro Rodrigues (Operário Rural), pela valiosa contribuição na condução deste trabalho.

\section{REFERÊNCIAS BIBLIOGRÁFICAS}

BAIN, D. C. Fungi recovered from seed of Sorghum vulgare Pers. Phytopathology, Saint Paul, v. 40, p. 521-522, 1950.

BRANCÃO, N.; SILVEIRA JÚNIOR, P.; CASELA, C. R.; MARTINS, R. M. Fungos da semente de sorgo (Sorghum bicolor) não controlados pelo tratamento com fungicidas. Fitopatologia Brasileira, Brasília, v. 8, n. 3, p. $575,1983$.

HARRIS, H. B.; LUTTRELL, E. S. Grain sorghum seed treatment tests and diseases in Georgia for 1954. Plant Disease Reporter, Beltsville, v. 39, p. 329-331, 1955.
HEPPERLY, P. R.; FELICIANO, C.; SOTOMAYOR, A. Chemical control of seedborne fungi of sorghum and their association with seed quality and germination in Puerto Rico. Plant Disease, Saint Paul, v. 66, n. 10, p. 902-904, Oct. 1982.

LASCA, C. C.; VECHIATO, M. H.; VALARINI, P. J. Detecção e identificação de fungos em sementes de sorgo (Sorghum sp.) produzidas no Estado de São Paulo. Arquivos do Instituto Biológico, São Paulo, v. 53, p. 47-54, 1986.

LIMONARD, T. A modified blotter test for seed health. Netherlands Journal of Plant Pathology, Wageningen, v. 72, p. 319-321, 1966.

MINUSSI, E. Taxonomia e esporulação de Colletotrichum graminicola (Ces.) Wils. (Sensu Arx. 1957) e patogenicidade em sorgo (Sorghum bicolor L.) Moench. 1977. 78 f. Tese (Doutorado) - Escola Superior de Agricultura Luiz de Queiroz, Piracicaba, 1977.

MINUSSI, E.; KIMATI, H. Alguns fungos sobre sementes de sorgo (Sorghum bicolor (L.) Moench. Revista do Centro Ciências Rurais, Santa Maria, v. 8, n. 4, p. 307-311, dez. 1978.

NEERGAARD, P. Seed pathology. 2. ed. London: Macmillan, 1979. $1191 \mathrm{p}$.

NOVO, R. J.; MENEZES, M. Eficiência de fungicidas no tratamento de sementes de sorgo granífero. Fitopatologia Brasileira, Viçosa, v. 9, n. 3, p. 543-549, 1984.

PINTO, N. F. J. A. Eficiência de fungicidas no tratamento de sementes de milho visando o controle de Fusarium moniliforme e Pythium sp. Pesquisa Agropecuária Brasileira, Brasília, v. 32, n. 8, p. 797-801, ago. 1997.

PINTO, N. F. J. A. Tratamento fungicida de sementes de sorgo visando o controle de fungos do solo e associados às sementes. Summa Phytopathologica, Jaboticabal, v. 24, n. 1, p. 26-29, jan./mar. 1998.

VALARINI, P. J.; LASCA, C. C.; VECHIATO, M. H.; SCHMIDT, J. R.; DION, P.; CHIBA, S. Tratamento de sementes de sorgo (Sorghum sp.) com fungicidas visando o controle de Colletotrichum graminicola (Ces,) Wils e outros fungos associados à sementes. Fitopatologia Brasileira, Viçosa, v. 13, n. 3, p. 238-243, out. 1988. 